$$
\text { "guncaga" — 2008/4/9 — 9:53 — page 349 — \#1 }
$$

\title{
Das Konzept des Analysisunterrichts von Professor Igor Kluvánek - einige Ergebnisse der qualitativen Forschung
}

\author{
JÁN GUNČAGA
}

\begin{abstract}
A renowned Slovak mathematician Professor Igor Kluvánek (1931-1993) during his affiliation with the University of Adelaide in Australia (1968-1990) has worked out a unique course of mathematical analysis for future high school teachers of mathematics. The course has been tested in its conceptual form but, as a whole, it still awaits its publication in the form of a monograph. Along these lines, our aim is to present the way he has introduced some key notions of differential calculus and to discuss its advantages. Central is the continuity of a function via which the limit and the derivative of a function at a point is defined.
\end{abstract}

Key words and phrases: Prof. Igor Kluvánek, mathematic analysis, continuity, limits and derivative of a function at a point.

ZDM Subject Classification: A30, I20, I40.

Professor Igor Kluvánek gehörte zu den bekanntesten slowakischen Mathematikern. Er wurde im Jahre 1931 in Košice geboren. Er studierte Vakuumtechnologie an der Elektrotechnischen Fakultät der Slowakischen Technischen Universität in Bratislava. In den Jahren 1952-1964 arbeitete er an dieser Universität am Lehrstuhl für Mathematik. In den Jahren 1964-1967 war er an der Naturwissenschaftlichen Fakultät der Universität von Pavol Jozef Šafárik in Košice tätig.

Nach 1950 hat Igor Kluvánek zusammen mit Ladislav Mišík und Marko Švec die Lehrbücher Mathematik I, II geschrieben. Sie wurden sehr bekannt und an

Copyright (C) 2007 by University of Debrecen 


$$
\text { "guncaga" — 2008/4/9 — 9:53 — page 350 — \#2 }
$$

der Hochschulen in der Tschechoslowakei bzw. in Tschechien und in der Slowakei mehr als 40 Jahren benutzt.

Während seines 23 jähriges Wirken (1967-1989) an der Flinders University in Adelaide entstand sein Gesamtwerk, das vor allem für die Lehramtstudenten bestimmt war. Nach dem Jahre 1989 kehrte Kluvánek in die Slowakei zurück, wo er 1993 verstarb.

\section{Stetigkeit einer Funktion an einer Stelle}

Dieses Thema wird entsprechend den Lehrplänen der slowakischen Gymnasien im letzten Studienjahrgang unterrichtet. Im Lehramtstudium der zukünftigen Lehrer gehört dieses Thema zum ersten Semester des Analysisunterrichtes. An der Pädagogischen Fakultät der Katholischen Universität in Ružomberok benutzen wir die Konzeption nach Professor Igor Kluvánek [5]. Andere Konzeptionen kann man sehen bei [1], [9], [4], [3], [10].

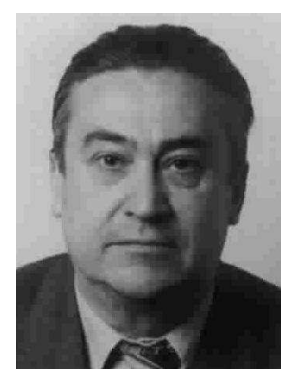

Abbildung 1. Igor Kluvánek (1931-1993)

Nach [6] hat es keinen Vorteil, wenn wir zuerst definieren die Grenzwert der Funktion und aus diesem Begriff die Stetigkeit der Funktion an einer Stelle. Es gibt keinen Unterschied von der logischen Seite, welchen Begriff wir nehmen als der erste. Es gibt aber einen grossen Unterschied von der pädagogischen Seite. Jeder erfahrene Lehrer betont, dass die Grenzwert der Funktion an einer Stelle keinen Funktionswert an dieser Stelle ist. Die Studenten haben nur geringe Erfahrungen mit diesen Begriffe und deshalb wahrnehmen sie diese Begriffe als identish.

Bei der Einführung der Grenzwert der Funktion an einer Stelle müssen wir lösen die Existenz diesen Begriff. Deshalb hat die Definition vier Quantoren. Definition der Stetigkeit ist leichter, weil sie nur drei Quantoren hat. Kluvánek in [6] 


$$
\text { "guncaga" — 2008/4/9 — 9:53 — page 351 — \#3 }
$$

hat den Begriff der Stetigkeit mit der Definition durch Umgebungen der Punkten eingeführt.

Wir haben im Herbst 2003 einen experimentalen Unterricht mit Studenten des letzten Jahrgangs des St. Andrea Gymnasiums in Ružomberok realisiert. Dieses Experiment war auf das Verständnis der Begriffe Stetigkeit, Grenzwert und Ableitung einer Funktion an einer Stelle orientiert.

Wir haben zuerst die Umgebung von einem Punkt eingeführt.

Lehrer: Wie können wir die Umgebung von einem Baum definieren?

Anna J.: Das ist ein Bereich, wo Blätter von diesem Baum fallen.

Lehrer: Interessante Definition. Wie sieht eine Viermeterumgebung von diesem Baum aus?

Anna J.: Kreis mit Radius 4.

Lehrer: Wenn wir haben einen Nummer auf dem Zahlengerade, können wir eine Umgebung der Zahl 5 finden?

Michal: Es wäre ein Intervall.

Lehrer: Wie sieht 0,5-Umgebung von der Zahl 5 aus?

Ján: Ein Intervall von 4,5 bis 5,5.

Lehrer: Richtig, es wäre das offene Intervall $(4,5 ; 5,5)$. Wie sieht $\varepsilon$ Umgebung der Zahl 5 aus?

L’ubomír: Das offene Intervall $(5-\varepsilon ; 5+\varepsilon)$.

Beispiel 1. Skizzieren sie den Graph der Funktion $y=2 x+5$.

Anna D. skizziert richtig den Graph der Funktion.

Lehrer: Wenn wir untersuchen den Graph dieser Funktion, sehen wir, dass die Funktionswerten aus der Umgebung der Zahl 3 in einer Umgebung der Zahl 11 sich befinden. Deshalb ist das Ungleichungssystem $10,5<2 x+5<11,5$ lösbar.

Michal:

$$
\begin{array}{rlrl}
10,5 & <2 x+5 & 2 x+5 & <11,5 \\
5,5 & <2 x & 2 x & <6,5 \\
2,75 & <x & x & <3,25
\end{array}
$$

Lehrer: Welche Umgebung zeigen mir diese Ungleichungen?

Lucia: Die Umgebung von der Zahl 3.

Die Studenten haben diesen Ergebnisse für jede Umgebung von der Zahl 11 verallgemeinert. 


$$
\text { "guncaga" — 2008/4/9 — 9:53 — page 352 — \#4 }
$$

Mária:

$$
\begin{array}{rlrl}
11-\varepsilon & <2 x+5 & 2 x+5 & <11+\varepsilon \\
6-\varepsilon & <2 x & 2 x & <6+\varepsilon \\
3-\frac{\varepsilon}{2} & <x & x & <3+\frac{\varepsilon}{2}
\end{array}
$$

Lehrer: Wir haben die $\frac{\varepsilon}{2}$-Umgebung von der Zahl 3 bekommen. Wenn wir bezeichnen $\delta=\frac{\varepsilon}{2}$, können wir für jede $\varepsilon$-Umgebung der Zahl 11 eine $\delta$-Umgebung der Zahl 3 mit dem Eigenschaft, dass Funktionswert in jedem Zahl aus dieser Umgebung in der $\varepsilon$-Umgebung der Zahl 11 sich befindet. Mária hat den Algorithmus benutzt, mit welchem wir für jede positive Realzahl $\varepsilon$ passende positive Realzahl $\delta$ finden. Wenn wir diesen Algorithmus umkehren, beweisen wir, dass für jede positive Realzahl $\varepsilon$ passende positive Realzahl $\delta$ existiert.

Nach dieser Lernsequenz war die Definition der Stetigkeit an einer Stelle und an einer Menge eingeführt. Die Studenten haben nächste Aufgaben gelöst.
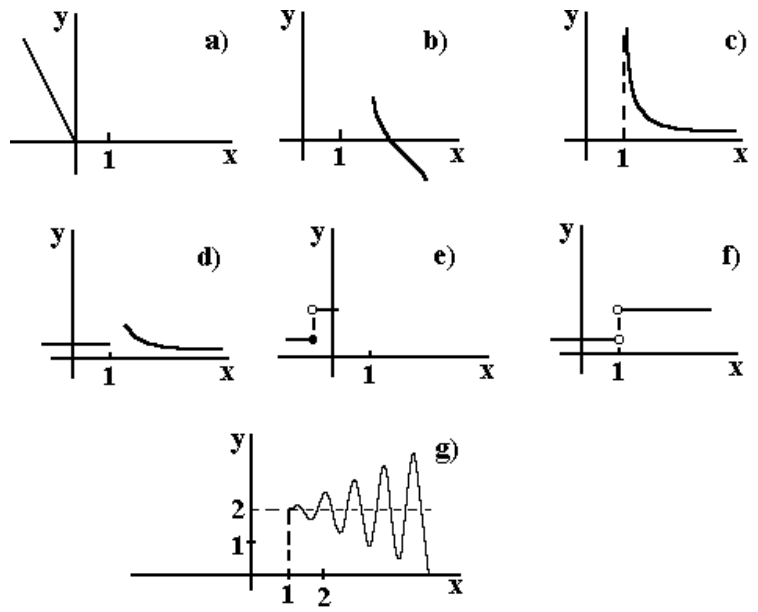

Abbildung 2

(1) Bestimmen Sie, welche aus nächsten Definitionen der Stetigkeit richtig sind.
A) $\forall \varepsilon>0 \exists \delta>0 \forall x \in D(f) ; a-\delta<x<a+\delta \Rightarrow f(a)-\varepsilon<f(x)<f(a)+\varepsilon$,
B) $\forall \varepsilon>0 \exists \delta>0 \forall x \in D(f) ; f(a)-\varepsilon<f(x)<f(a)+\varepsilon \Rightarrow a-\delta<x<a+\delta$,
C) $\forall \varepsilon>0 \exists \delta>0 \forall x \in D(f) ; a-\varepsilon<x<a+\varepsilon \Rightarrow f(a)-\delta<f(x)<f(a)+\delta$,
D) $\forall \delta>0 \exists \varepsilon>0 \forall x \in D(f) ; f(a)-\varepsilon<f(x)<f(a)+\varepsilon \Rightarrow a-\delta<x<a+\delta$. 


$$
\text { "guncaga" — 2008/4/9 — 9:53 — page 353 — \#5 }
$$

(2) Beenden Sie nächste Graphen der Funktionen, damit diese Funktionen stetig an der Stelle 1 werden (siehe Abbildung 2).

(3) Welche Funktionen mit den Graphen gegeben stetig an der Stelle 1 sind?
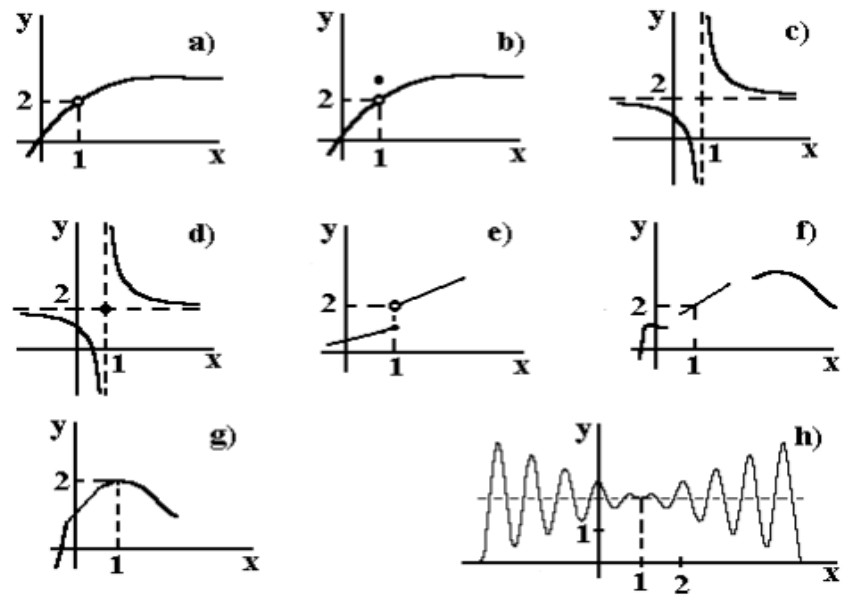

Abbildung 3

Zwei Drittel der Studenten haben die Aufgabe 1 richtig gelöst. Einige Studenten haben die Möglichkeiten B und D gewählt, weil sie Probleme mit der Folge der Quantoren und der Folge der Aussagen in der Implikation hatten.

In der Aufgabe 2 hat niemand alle Graphen richtig skizziert. Die Fehler waren meistens in den Graphen $b, d$ und $e$. Bei den Graphen $d$ und $e$ haben die Studenten geantwortet, dass es unmöglich ist, diese Graphen zu beenden. Einige Studenten haben im Graph $d$ eine Arm der Hyperbel skizziert.

Die Aufgabe 3 hat eine Hälfte der Schüler richtig gelöst. Die falsche Antworten waren meistens, dass die Funktionen $b, d$ und $e$ stetig sind und die Funktion $f$ ist unstetig an der Stelle 1. Die Studenten hatten eine falsche Meinung, dass wenn eine Funktion an einer Stelle definiert ist, dann ist diese Funktion stetig an dieser Stelle. Die Funktion $f$ war definiert nur in einer Umgebung der Zahl 1 und das war ein Problem für Studenten. 
认

\section{LIMITS}

The notion of the limit of a function at a point is losely related to the idea of continuity. If we can choove that the function becomes continuows at that foint, then the number is called the linit of the given function at the given point. In this chapter we study birefly this notion

\section{The definition}

Suppose we are given a function defined at every point of a neighbourhood of a point $a$ with the possible exception of the point a itself. We may try to find a number $k$ such that, if it is declared to be the value of the given function at $a$, then the function becomes continuous at $a$. Such a number $k$ is then called the limit of the given function at the point $a$.

Let us state the definition of limit more clearly and precisely.

Given a function $f$, a point $a$ and a number $k$, let $F$ be the function such that

(i) $E(x)=f(x)$, for every $x \neq a$ in the domain of the function $f$; and

(ii) $F(a)=k$.

The value of the function $F$ is the same as the value of $f$ at every point of the domain of the function $f$ except, possibly, at $a$. However, the point $a$ may not belong to the domain of $f$ or the value $f(a)$ may not be equal to $k=F(a)$, therefore the functions $F$ and $f$ are not necessarily equal to each other.

The problem is to choose the number $k$ so that the function $F$ becomes continuous (left-continuous, right-continuous) at $a$. To be sure, such a choice may not be possible. However, the principle of continuous extension (Theorem $I$. 9.1) says that, if there exists a number $k$ such that the function $F$ is continuous (left-continuous, right-continuous) at $a$, then it is unique

Now we formally state the definition of limit.

The limit (left limit, right limit) of a function $f$ at a point $a$ is the number $k$ such that the function $F$, defined by the requirements (i) and (ii) is continuous (left-continuous, right-continuous, respectively) at $a$.

$$
\begin{aligned}
& \text { Example 1. Let } \\
& \qquad f(x)=\frac{x^{2}-9}{x-3} \\
& \text { for everg } x \neq 3 \text {. The nunter } 6 \text { is the limit of the function } f
\end{aligned}
$$

Abbildung 4. Eine Teil der Handschriften von Professor Igor Kluvánek 


$$
\text { "guncaga" — 2008/4/9 — 9:53 — page 355 — \#7 }
$$

\section{Grenzwert der Funktion an einer Stelle}

Der Grenzwert der Funktion $f$ an der Stelle $a$ ist ähnlich wie bei Königsberger [7] und Pickert [8] mit dem Begriff der stetigen Fortsetzung der Funktion $f$ an der Stelle $a$ verbunden.

Man sieht es in dieser Definition:

Definition 1. Sei die Funktion $f$ definiert in einer Umgebung $W$ der Stelle $a$. Die Funktion $f$ hat an der Stelle a den (rechtsseitigen, linksseitigen) Grenzwert L, wenn die Funktion $F$ :

(1) $F(x)=f(x)$ für jedes $x \in W$ und $x \neq a$,

(2) $F(x)=L$ für $x=a$

(rechtsseitig, linksseitig) stetig an der Stelle $a$ ist. Man schreibt:

$$
\left(\lim _{x \rightarrow a^{+}} f(x)=L, \lim _{x \rightarrow a^{-}} f(x)=L\right) \lim _{x \rightarrow a} f(x)=L .
$$

Die Benutzung dieser Definition zeigen wir an einem Beispiel:

Beispiel 2. Zeigen Sie, dass die Funktion $f(x)=\frac{x^{2}-1}{x+1}$ an der Stelle 1 einen Grenzwert hat.

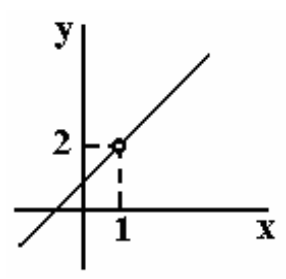

Abbildung 5

Lösung. Die Funktion $F(x)$ ist für $x \neq a: F(x)=\frac{x^{2}-1}{x+1}=x+1$. Wenn wir wollen, dass die Funktion $F(x)$ stetig an der Stelle 1 wird, definieren wir $F(1)=2$.

Deshalb hat die Funktion $f(x)=\frac{x^{2}-1}{x+1}$ an der Stelle 1 den Grenzwert 2 und wir können schreiben: $\lim _{x \rightarrow 1} \frac{x^{2}-1}{x-1}=2$. 
Diesen Begriff haben wir in unserem experimentalen Unterricht auf nächsten Problemaufgaben geübt.

(1) Wir wissen, dass es für die Funktion $f$ gilt: $\lim _{x \rightarrow 1} f(x)=2$. Welche Funktion von den nächsten Funktionen (mit den Graphen gegeben) erfüllen diese Bedingung? Begründen Sie Ihren Antwort für jede Funktion (siehe Abbildung $3)$.

(2) Finden Sie eine positive reelle Zahl $\delta$, welche die nächste Bedingung erfüllt.

(a) $3-\delta<x<3+\delta \Rightarrow 0,9<\frac{1}{x-2}<1,1$;

(b) $2-\delta<x<2+\delta \Rightarrow-10<\frac{1}{x-2}<10$.

Die ganze Aufgabe 1 hat niemand richtig gelöst. Eine Hälfte der Studenten gaben den Antwort, dass die Funktion a) die Bedingung erfüllt. Bei der Funktion b) gab es nur drei Studenten. Die Studenten haben nicht verstanden, dass wenn die Funktion nicht stetig an einer Stelle ist und sie hat einen Funktionswert an dieser Stelle, dann kann diese Funktion einen Grenzwert an dieser Stelle haben. Eine Viertel der Studenten gabe den falschen Antwort, dass die Funktion c) die Bedingung erfüllt. Ein Student dachte, dass die Funktion d) nicht stetig ist. Ein Viertel der Studenten hat bei den Funktionen f), g), h) geschrieben, dass diese Funktionen stetig an der Stelle 1 sind und sie haben die Begriffe Grenzwert und Stetigkeit verwechselt. Drei Viertel der Studenten war der Meinung, dass die Funktion h) keinen Grenzwert an der Stelle 1 hat und einige Studenten hatten die Meinung, dass diese Funktion nicht stetig ist.

Die Aufgabe 2a hat über eine Hälfte der Studenten richtig gelöst. Zwei Studenten hatten falsche Lösungen mit nummerischen Fehlern und andere Studenten haben diese Aufgabe nicht gelöst. Die Aufgabe 2b hat eine Hälfte der Studenten richtig gelöst. Drei Studenten hatten falsche Lösungen mit nummerischen Fehlern und andere Studenten haben diese Aufgabe nicht gelöst. In den falschen Lösungen waren auch die Fehler bei der Multiplikation der Ungleichung.

\section{Die Ableitung}

Auch die Ableitung der Funktion $f$ an der Stelle $a$ steht mit dem Begriff der stetigen Fortsetzung der Funktion $f$ an der Stelle $a$ in Beziehung. Kluvánek definiert zuerst die Differenzierbarkeit einer Funktion und dann die Ableitung:

Definition 2. Sei die Funktion $f$ definiert in einer Umgebung $W$ der Stelle $a$. Die Funktion $f$ ist differenzierbar an der Stelle $a$, wenn eine Funktion $\varphi(u)$ 
existiert, die stetig an der Stelle 0 ist und die Eigenschaft: $f(a+u)-f(a)=$ $\varphi(u) \cdot u, a+u \in W$ hat.

Den Wert $\varphi(0)$ nennen wir die Ableitung der Funktion $f$ an der Stelle a und bezeichnen ihn mit $f^{\prime}(a)$.

Diese Definition hat viele Anwendungen in der Praxis. Zum Beispiel: wenn die Funktion $f(x)$ die Wegstrecke eines Körpers im Zeitpunkt $x$ ist, dann ist die Funktion $\varphi(u)$ die Durchschnittsgeschwindigkeit dieses Körpers zwischen den Zeitpunkten $a$ und $a+u$. Der Wert $\varphi(0)$, d. h. die Ableitung der Funktion $f$ an der Stelle $a$, ist dann die Momentangeschwindigkeit dieses Körpers im Zeitpunkt $a$.

Diese Definition hat auch Vorteile beim Verstehen, wann die Ableitung der Funktion $f$ an der Stelle $a$ existiert und wann nicht. Das zeigen wir an den folgenden zwei Beispielen:

Beispiel 3. Sei $f(x)=x^{3}$. Wie findet man $f^{\prime}(2)$ ?

Lösung. Die Funktion $f(x)$ ist definiert für jede reelle Zahl $x$. Wir benutzen die Funktion $f(2+u)-f(2)=\varphi(u) \cdot u$. Wir bekommen:

$$
\begin{aligned}
(2+u)^{3}-2^{3} & =\varphi(u) \cdot u \\
8+12 u+6 u^{2}+u^{3}-8 & =\varphi(u) \cdot u \\
u\left(12+6 u+u^{2}\right) & =\varphi(u) \cdot u \\
12+6 u+u^{2} & =\varphi(u)
\end{aligned}
$$

Die Funktion $\varphi(u)=12+6 u+u^{2}$ ist eine quadratische Funktion.

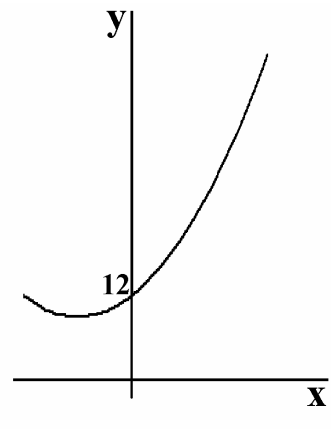

Abbildung 6

Deshalb ist sie stetig an der Stelle 0 mit dem Funktionswert $\varphi(0)=12$. Also gilt $f^{\prime}(2)=\varphi(0)=12$. 
Beispiel 4. Sei $g(x)=|x-2|$. Existiert $g^{\prime}(2)$ ?

Lösung. Die Funktion $g(x)$ ist definiert für jede reelle Zahl $x$. Wir benutzen die Funktion $g(2+u)-g(2)=\varphi(u) \cdot u$. Wir bekommen:

$$
\begin{aligned}
|2+u-2|-|2-2| & =\varphi(u) \cdot u \\
|u| & =\varphi(u) \cdot u \\
\frac{|u|}{u} & =\varphi(u) \text { für jedes } u \neq 0 .
\end{aligned}
$$

Deshalb ist $\varphi(u)=1$ für jedes $u>0$ und $\varphi(u)=-1$ für jedes $u<0$.

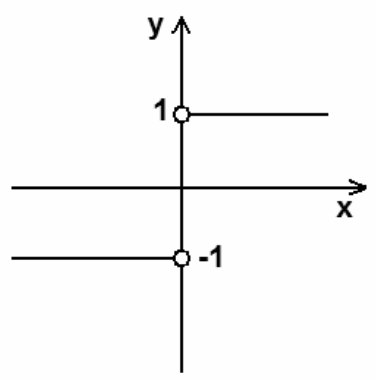

Abbildung 7

$\varphi(u)$ ist nicht stetig an der Stelle 0. Wir können keinen Funktionswert $\varphi(0)$ definieren, mit welchem diese Funktion stetig wäre. $g^{\prime}(2)$ existiert nicht.

Im experimentalen Unterricht haben wir diesen Beispiel auf nächsten Funktion verallgemeinert.

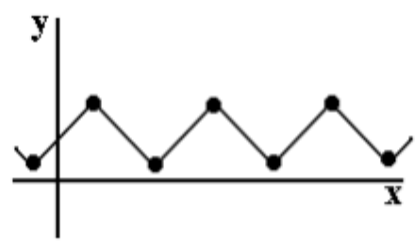

Abbildung 8

Wenn wir die Ableitung einer linearen Funktion gerechnet haben, haben die Studenten die nächste Aufgabe gelöst: 


$$
\text { "guncaga" — 2008/4/9 — 9:53 — page } 359 \text { — \#11 }
$$

Es gilt, dass $f^{\prime}(3)=2$. Welche Funktion aus den Funktionen von a) bis f) diese Bedingung erfüllt?
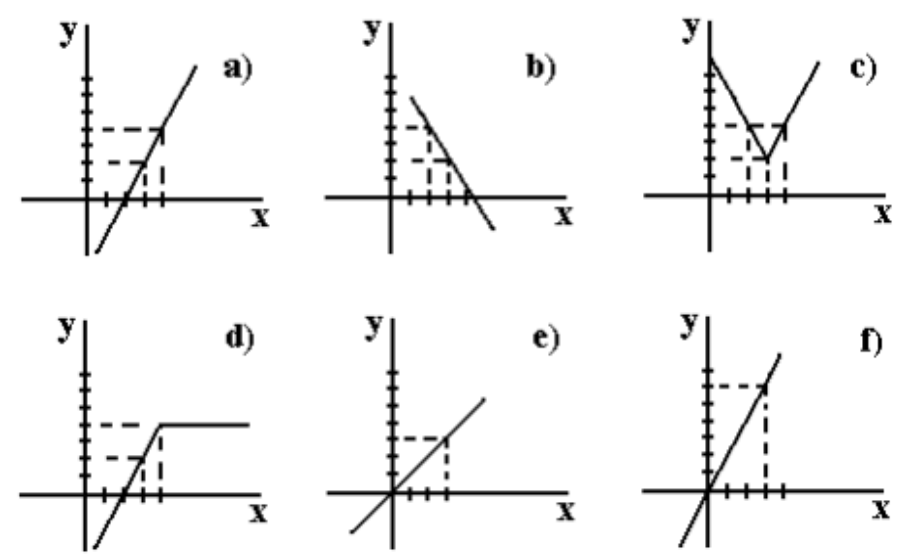

Abbildung 9

Ganze Aufgabe hat weniger als eine Viertel der Studenten richtig gelöst. Die meisten falschen Antworten waren die Funktionen b) und d).

\section{Schlussbemerkungen}

Analysisunterricht ist oft mehr orientiert auf die Lösung der kalkulativen Aufgaben und weniger auf die Aufgaben, die auf das Begriffverständniss orientiert sind. Hier kann uns die Konzeption von Professor Igor Kluvánek helfen.

Vorteile der Konzeption von Professor Igor Kluvánek liegen darin, dass die Studenten die Grundbegriffe der Analysis tiefer verstehen und mehr mit graphischen Darstellungen der Funktionen arbeiten können. Die Begriffe „Grenzwert einer Funktion an einer Stelle“ und „Ableitung einer Funktion an einer Stelle“ sind durch die Stetigkeit definiert und diese Definitionen sind für Studenten leichter als die Definitionen durch den Grenzwert.

Viele Studenten verwechseln die Begriffe „Grenzwert einer Funktion an einer Stelle" und "Stetigkeit einer Funktion an einer Stelle". Hier ist es wichtig, im Unterricht Beispiele von Funktionen zu verwenden und aufzuzeigen, die an der betreffenden Stelle 
- einen Grenzwert an der Stelle haben und an dieser Stelle auch stetig sind,

- einen Grenzwert an der Stelle haben und an dieser Stelle nicht stetig sind,

- keinen Grenzwert an der Stelle haben (siehe [2]).

Die Benutzung den Graphen der Funktionen kann sehr helfen bei der Einführung des Begriffs Ableitung der Funktion an einer Stelle. Die Schüler können besser und visuell verstehen den Unterschied zwischen der Existenz und die Nichtexistenz der Ableitung der Funktion an der Stelle. Es gibt die Möglichkeit diese Konzeption des Analysisunterrichts in den Naturwissenschaften anwenden (zum Beispiel in der Physik).

Die Studenten brauchen im Analysisunterricht die Kenntnisse aus anderen Bereichen der Schulmathematik (Ungleichungen, algebraische Terme, usw.) Wenn diese Kenntnisse fehlen, das hat eine negative Auswirkung auf die Leistungen der Studenten bei der Lösung der Aufgaben.

Bemerkung. Dieser Beitrag wurde unterstützt vom Grant KEGA 3/3269/05 und vom Projekt ESF 11230220297.

\section{Literatur}

[1] S. Domoradzki, Stefan Banach's textbooks, in: Matematika v škole dnes a zajtra, PF KU, Ružomberok, 2006, 46-50.

[2] J. Gunčaga, Grenzwertprozesse in der Schulmathematik, Journal für MathematikDidaktik, Heft 1, Stuttgart - Leipzig - Wiesbaden (2006), 77-78.

[3] J. Gunčaga, Limitné procesy v školskej matematike (Grenzwertprozesse in der Schulmathematik), Dissertation, FPV UKF, Nitra, 2004, http://fedu.ku.sk/〜guncaga/publikacie/DizWeb.pdf.

[4] P. Eisenmann, Test des infinitesimalen Denkens, Mathematica didactica, Band 1, Hildesheim (2000), 63-71.

[5] I. Kluvánek, Handschriften zur Differential- und Integralrechnung.

[6] I. Kluvánek, Čo nie je dobré vo vyučovaní matematickej analýzy? (Was ist nicht gut im Analysisunterricht?), Matematické obzory 36 (1991), 23-49, 37, 47-66.

[7] B. Königsberger, Analysis I, Springer Verlag, Berlin-Heidelberg-New York, 2001.

[8] G. Pickert, Aufbau der Analysis vom Stetigkeitsbegriff her, Der Mathematische und naturwissenschaftliche Unterricht, Band 21, Heft 11 (1968), 384-388.

[9] ك̌. Tkačik, Why Logarithms?, in: XIV th Czech-Polish-Slovak Mathematical School, Czestochowa, 2007, 429-434.

[10] E. Wachnicki, Z. Powązka, Problemy analizy matematycznej w zadaniach. Cęść I (Die Probleme des Analysisunterrichts in den Aufgaben. Teil I), AP, Kraków, 2002. 


$$
\text { "guncaga" — 2008/4/9 — 9:53 — page 361 — \#13 }
$$

JÁN GUNČAGA

LEHRSTUHL FÜR MATHEMATIK

PÄDAGOGISCHE FAKULTÄT DER KATHOLISCHEN UNIVERSITÄT

NÁMESTIE ANDREJA HLINKU 56

03401 RUŽOMBEROK

SLOWAKEI

E-mail: guncaga@fedu.ku.sk

(Received May, 2007) 